\author{
Strange Fruit \\ Designer: Sophia Luu, Iowa State University, USA \\ Mentor: Ling Zhang, Iowa State University, USA
}

Keywords: digital printing, engineered print, hand crafts

\title{
Mentor Statement
}

Strange Fruit was created in an experimental design process course for undergraduate students. The purpose of this mentorship relationship was for the students to explore and experiment with digital textile printing and social issues through an intensive design process to create a marketable piece. The goal of this junior-level course was to understand the design process of digital textile printing and research concept in apparel design. As design mentor, my role was to demonstrate the print pattern development, techniques of engineered prints, and hand beading. I also guided the student through the entire design process, provide the necessary resources, and advise recommendations for the design. The challenge of this design was to develop the prints that present an awareness of sexual violence as a weapon of war, and then apply this concept onto the garment patterns. In this project, the student was struggling to connect the prints on the front and back jacket. I suggested that she continue the service metal patterns to the back piece and add hand embroidery to emphasize the small color blocks. I chose to submit this work, because it exhibits the benefit of utilizing digital textile printing technology and a high level of creativity in the incorporation of social issues of sexual violence in achieving an art-to-wear design.

\section{Design Statement}

The purpose of Strange Fruit was to create an ensemble bringing awareness to sexual violence as a weapon of war through a digitally engineered print design and the use of a militaristic silhouette. The designer was inspired by their visit to the Nobel Peace Center in Oslo, Norway, where the current exhibition was "The Body as a Battlefield." This exhibition was dispayed in part because of the 2019 Nobel Peace Prize Laureates Denis Mukwege and Nadia Murad, who were "awarded for their efforts to end the use of sexual violence as a weapon of war and armed conflict" (Sleire, 2018). Photography by Cristina De Middel was included in the exhibition. It was from her photography that the engineered print design was most inspired.

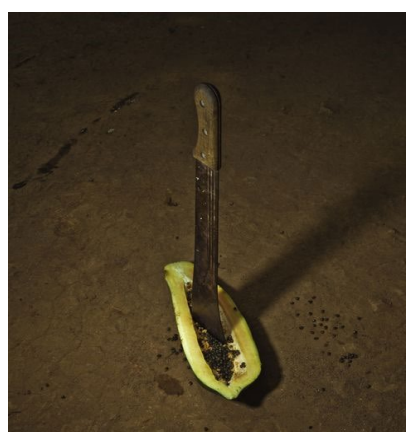

Figure 1: Photograph from "The Body as a Battlefield" exhibition.

Similar design work includes Dr. Kelly Reddy-Best's "The Emotions of Rape," which "brings awareness to the amount of unreported rapes that occur in the United States" (Reddy-Best, 2013). Dolce and Gabanna's 2016 Forbidden Fruit is similar with its large, engineered fruit spot print, and Versace's Spring 2019 RTW collection is similar with its militaristic silhouettes. The

Page 1 of 3

(c) 2020 The author(s). Published under a Creative Commons Attribution License

(https://creativecommons.org/licenses/by/4.0/), which permits unrestricted use, distribution, and reproduction in any medium, provided the original work is properly cited.

ITAA Proceedings, \#77 - https://itaaonline.org 
most inspiring photo for the designer was Middel's photograph of a papaya with a machete. It is symbolic of a woman's womb attacked by sexual violence. In relation to war, the designer was intrigued by service medals with their bright colors and linear design.

The process began with the draping of a jacket. Princess style lines were utilized to avoid issues with darts in the engineered print process. A mandarin collar was utilized because of its similarity to military uniforms and an asymmetrical front fit for hidden snap closures was developed. Upon pattern creation and construction of a muslin mockup, the pattern was digitized and exported to Adobe Illustrator and then to Adobe Photoshop to begin the engineering of the prints. Shorts were flat patterned, but did not feature any digital print design.

For the creation of the print design, the designer took primary photographs of a sliced papaya and papaya seeds. These photographs were loaded into Adobe Photoshop, adjusted with the red boost filter for vibrancy, and cut symmetrically for use on the facing of the jacket. Seed images were made into multi-layered Photoshop files for ease in placement of the papaya seeds in the engineered print design.

A service medal decoration was created by the designer in Adobe Illustrator using the rectangle tool. Rectangles were created proportionately to the dimensions of realistic service medals and the aesthetic desired by the designer. The rectangles were then grouped and made into a repeat pattern design. The designer filled all pattern pieces with a black color to begin with in Adobe Photoshop (Figure 2). Regarding the papaya, the designer placed the right side of the papaya on the left center front panel, then utilized the lasso tool to cut away, so the papaya went out of the bounds of the pattern piece. Since the pattern piece was slightly rounded being on a princess style line, the designer used the puppet warp tool to make the papaya fit the final sewing dimensions. The papaya was split onto the left side panel and left front facing as well. With the service medals, the designer resized the Adobe Illustrator graphic to cover the span of pieces in Adobe Photoshop. The designer situated the medal perpendicular to the grain and utilized the layers panel to place



Figure 2: Engineered prints on garment patterns. the service medals on the front right facing, front right center panel, front right-side panel, and back right-side panel. For the back-pattern pieces, medal motifs were individually placed in layers to enable the designer to create a fading effect with the medals. Papaya seeds were placed in individual layers as well so the designer could individually place each seed. Upon completion of the print panels, layers were compressed and the file was exported as a high-quality JPEG.

Page 2 of 3

(c) 2020 The author(s). Published under a Creative Commons Attribution License (https://creativecommons.org/licenses/by/4.0/), which permits unrestricted use, distribution, and reproduction in any medium, provided the original work is properly cited.

ITAA Proceedings, \#77 - https://itaaonline.org 


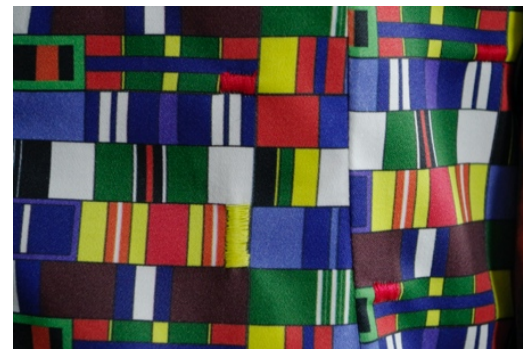

Figure 3: Zig zag stitching on service medals.
The fabric was printed on cotton sateen for its smooth hand and structural weight. During garment construction the designer also incorporated surface design through zig zag stitching on a home sewing machine and via hand beading. A few service medals were highlighted throughout with similarly colored thread such as red, yellow, green, and blue (Figure 3). The black shorts also featured a fading service medal motif through this zig zag stitch application. In the white, glinted areas of the papaya, white seed beads were applied. To accent the subtle papaya seeds, black seed beads were applied.

Activist fashion design has rarely touched upon the topic of sexual violence as a weapon of war. By merging engineered print design with a rare activist subject matter, Strange Fruit makes an innovative contribution as an art-to-wear ensemble.

References:

Reddy-Best, K. (2013). The Emotions of Rape. International Textile and Apparel Association (ITAA) Annual Conference Proceedings.

Sleire, S. (2018, October 5). Denis Mukwege and Nadia Murad Awarded Nobel Peace Prize. Retrieved December 16, 2019, from https:/www.bloomberg.com/news/articles/2018-1005/denis-mukwege-and-nadia-murad-awarded-nobel-peace-prize. 

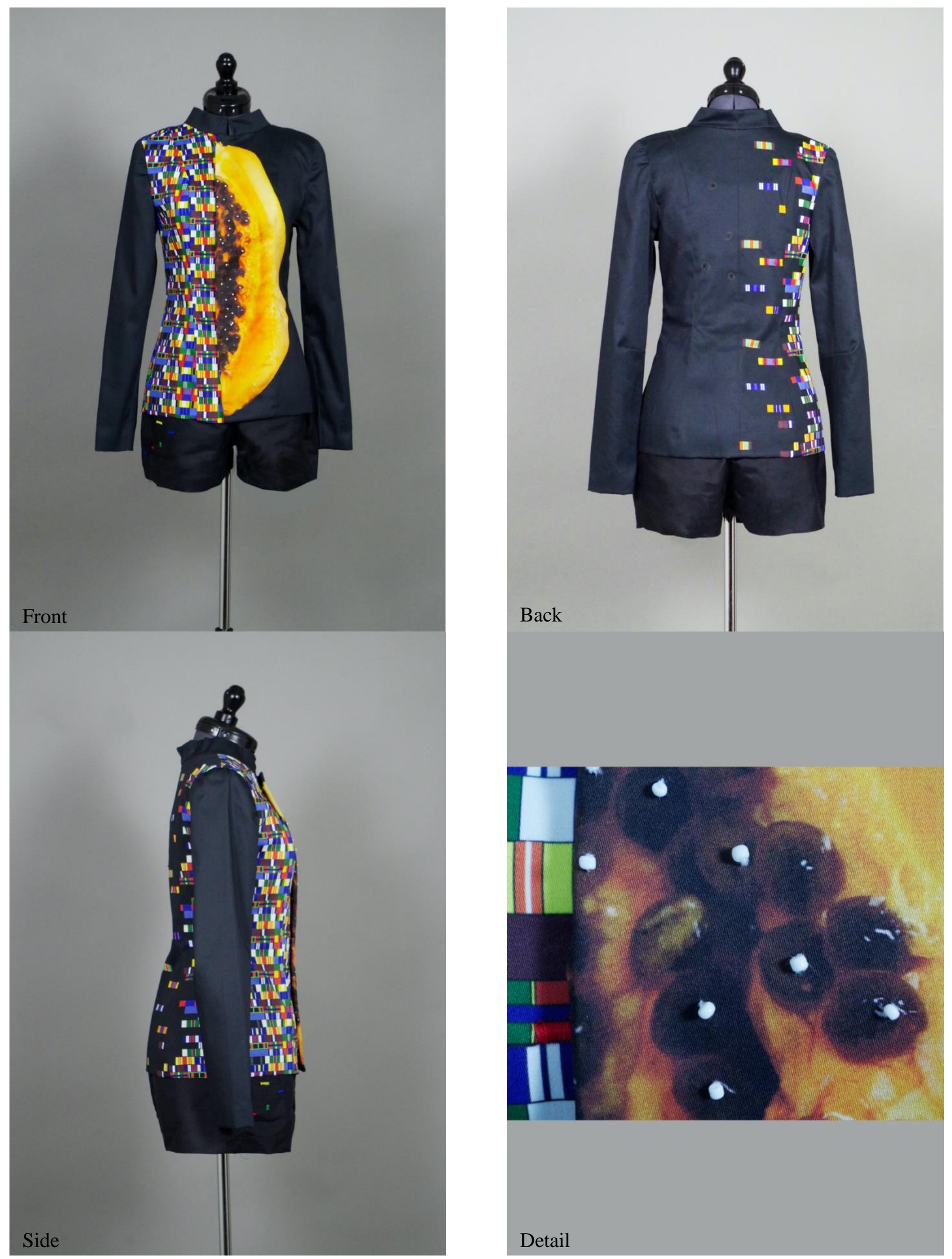